\title{
A Methodology for identifying and improving occupant behavior in residential buildings
}

\author{
Zhun (Jerry) Yu ${ }^{1}$, Fariborz Haghighat ${ }^{1}$, Benjamin C. M. Fung ${ }^{2}$, Edward Morofsky $^{3}$, \\ Hiroshi Yoshino ${ }^{4}$ \\ ${ }^{1}$ Department of Building, Civil and Environmental Engineering, Concordia University, \\ Montreal, Quebec, H3G 1M8, Canada \\ ${ }^{2}$ Concordia Institute for Information Systems Engineering, Concordia University, \\ Montreal, Quebec, H3G 1M8, Canada \\ ${ }^{3}$ Real Property Branch, Public Works and Government Services Canada, Place du Portage III, \\ 8B1, Gatineau, Québec, K1A 0S5 Canada \\ ${ }^{4}$ Department of Architecture and Building Science, Tohoku University, Japan
}

\begin{abstract}
This paper reports the development of a methodology for identifying and improving occupant behavior in existing residential buildings. In this study, end-use loads were divided into two levels (i.e. main and sub-category), and they were used to deduce corresponding two-level user activities (i.e. general and specific occupant behavior) indirectly. The proposed method is based on three basic data mining techniques: cluster analysis, classification analysis, and association rules mining. Cluster analysis and classification analysis are combined to analyze the main end-use loads, so as to identify energy-inefficient general occupant behavior. Then, association rules are mined to examine end-use loads at both levels, so as to identify energy-inefficient specific occupant behavior. In order to demonstrate its applicability, this methodology was applied to a group of residential buildings in Japan, and one building with the most comprehensive household appliances was selected as the case building. The results show that, for the case building, the method was able to identify the behavior which needs to be modified, and provide occupants with feasible recommendations so that they can make required decisions. Also, a reference building can be identified for the case building to evaluate its energy-saving potential due to occupant behavior modification. The results obtained could help building occupants to modify their behavior, thereby significantly reducing building energy consumption. Moreover, given that the proposed method is partly based on the comparison with similar buildings, it could motivate building occupants to modify their behavior.
\end{abstract}

Keywords: Occupant behavior; Building energy consumption; Data mining; Evaluation; Identification 
Nomenclature

$\begin{array}{ll}\text { SHW } & \text { Supply hot water load } \\ \text { LIGHT } & \text { Lighting load } \\ \text { KITCH } & \text { Kitchen load } \\ \text { REFRI } & \text { Refrigeration load } \\ \text { E\&I } & \text { Entertainment \& Information load } \\ \text { H\&S } & \text { Housework \& Sanitary load } \\ \text { OTHER } & \text { Others load } \\ \text { T } & \text { Outdoor temperature (annual average) }\left({ }^{\circ} \mathrm{C}\right) \\ \text { RH } & \text { Outdoor relative humidity }(\text { annual average }) \\ \text { V } & \text { Outdoor air velocity }(\text { annual average })(\mathrm{m} / \mathrm{s}) \\ \text { RA } & \text { Outdoor solar radiation }(\text { annual average })\left(\mathrm{MJ} / \mathrm{m}^{2}\right) \\ \text { NO } & \text { Number of occupants } \\ \text { FA } & \text { Floor area }\left(\mathrm{m}^{2}\right) \\ \text { HLC } & \text { Heat loss coefficient }\left(\mathrm{W} / \mathrm{m}^{2} \mathrm{~K}\right) \\ \text { ELA } & \text { Equivalent leakage area }\left(\mathrm{cm}^{2} / \mathrm{m}^{2}\right) \\ \text { CO } & \text { Construction } \\ \text { SH } & \text { Space heating } \\ \text { WH } & \text { Water heating } \\ \text { KIT } & \text { Kitchen } \\ \text { HT } & \text { House type } \\ & \end{array}$




\section{Introduction}

Currently, residential sector building energy consumption forms a large part of the total national energy consumption (TNEC) in both developed and developing countries. For example, in the US and Japan, residential building energy consumption accounts for $25 \%$ and $26 \%$ of TNEC, respectively [1]. In China and Thailand, the proportion of residential building energy consumption to TNEC is $11.3 \%$ and $15.4 \%$, respectively [2-3]. Furthermore, with the rapid growth of the economy and rising living standards, there is a rapid increase in energy consumption in the residential sector worldwide [4-6]. The high energy demand in residential buildings, which is also growing rapidly, necessitates a better understanding of its major influence factors. At the same time, it is necessary to develop a methodology for reducing energy consumption. For instance, to combat this rapid increase in energy use some utility companies and government organizations provide building owners with a "booklet" which gives tips on reducing the building energy consumption. These tips are general in nature and are not specific.

Among various factors influencing residential building energy consumption, occupant behavior plays an essential role and is difficult to investigate analytically due to its complicated characteristics [7]. Note that here occupant behavior refers to activities that have a direct or indirect impact upon building energy consumption. For example, occupants turn on/off lights, TV sets, computers, microwave ovens, and so on. Commonly such behavior is associated with various household appliances and thus can be deduced indirectly from corresponding end-use loads. For example, the total daily (or monthly, annual) lighting energy consumption in a residential building qualitatively indicates the duration of lighting usage in this day (or month, year). Accordingly, any improvement in the occupant behavior leads to the reduction of the residential building energy consumption.

Recently, there has been increasing interest in studying occupant behavior and developing a methodology for identifying the corresponding energy-saving potential. Ouyang and Hokao [8] investigated the energy-saving potential by improving user behavior in 124 households in China. In this study, these houses were divided into two groups: one group received an energy-saving education and was encouraged to put energy-conscious behavior into effect, while the other group was required to keep behavior intact. Comparisons were made between monthly household electricity uses in July 2007 and July 2008 for both groups. It was found that, on average, effective promotion of energy-conscious behavior could reduce household electricity consumption by more than $10 \%$. Al-Mumin et al. [9] simulated occupant behavior improvement (i.e. simulation of occupant behaviour before and after modification) and corresponding annual electricity consumption reduction by using the energy simulation program ENERWIN. They first collected data and information on occupancy patterns and operation schedules of electrical appliances in 30 selected residences in Kuwait. This data and information were then used in ENERWIN to 
replace the default value. A house then was selected as a case study and the simulation results showed that the annual electricity consumption in this house was increased by 21\%. The results also indicated that the ENERWIN's default parameters (i.e. parameters taken from the software manual) are probably more appropriate for the Western living lifestyle. Moreover, it was found that a reduction of energy consumption by $39 \%$ can be achieved by improving occupant behavior such as turning off the lights when rooms were empty and setting the air conditioner thermostat to a higher temperature (but still within the comfort level).

Basically, two approaches (i.e., energy-saving education and building simulation), were used to improve building occupant behavior and identify the corresponding energy-saving potential. These two approaches can help to modify occupant behavior and have an immediate effect on building energy consumption reduction. However, both of the approaches have certain limitations. With regard to the energy-saving education approach, commonly detailed energy-saving measures and tips on efficient use of various household appliances should be provided for occupants. Considering that a family normally has a number of appliances and that each appliance may have various tips (e.g. for the usage of refrigerators, various tips can be given: reduce door open times, keep its coils and filters clean, keep it far from other heat sources,etc), there could be a large number of energy-saving measures and tips for an individual family. For example, one family may have 30 household appliances, with each appliance having an average of 8 energy-saving tips. Accordingly, the occupants need to understand and remember 240 tips, which may be quite impractical. Although a booklet of these tips can be prepared for building occupants, it is very difficult for occupants to remember distinctly all these tips and implement them for a long time in practice. Furthermore, occupants may not fully understand and have confidence in these tips' effects as they only provide qualitative information. In addition, some energy-saving opportunities can only be initiated by building occupants. For example, when occupants realize they have consumed too much energy on both computers and TVs, they can avoid using both devices simultaneously when they can only focus on one of them, or make a conscious effort to reduce usage time.. Therefore, instead of simply providing occupants with a number of general energy-saving recommendations, it is more rational and efficient to help them modify the behaviour in two steps. First, it is necessary to identify the behaviour that needs to be modified. This can be achieved by analyzing measured data. Second, feasible recommendations to improve the identified behaviour can be presented with the goal of reducing energy consumption in the home. With regard to the building simulation approach, current simulation tools can only imitate some typical activities such as the control of sun-shading devices in a rigid way, while realistic building occupant behavior patterns are more complicated.

This paper reports the development of a rational methodology for identifying and improving occupant behavior in existing residential buildings, based on an analysis of collected data and information. In particular, feasible recommendations are made for 
assisting occupants to modify their behaviour so as to reduce energy consumption.

\section{Methodology}

A new methodology is proposed for efficiently improving occupant behavior in existing residential buildings, and evaluating the energy-saving potential resulting from these modifications. As mentioned previously, end-use loads are used to deduce user activities indirectly. Specifically, these loads are used to map onto occupant behavior at two levels, as shown in Fig. 1.

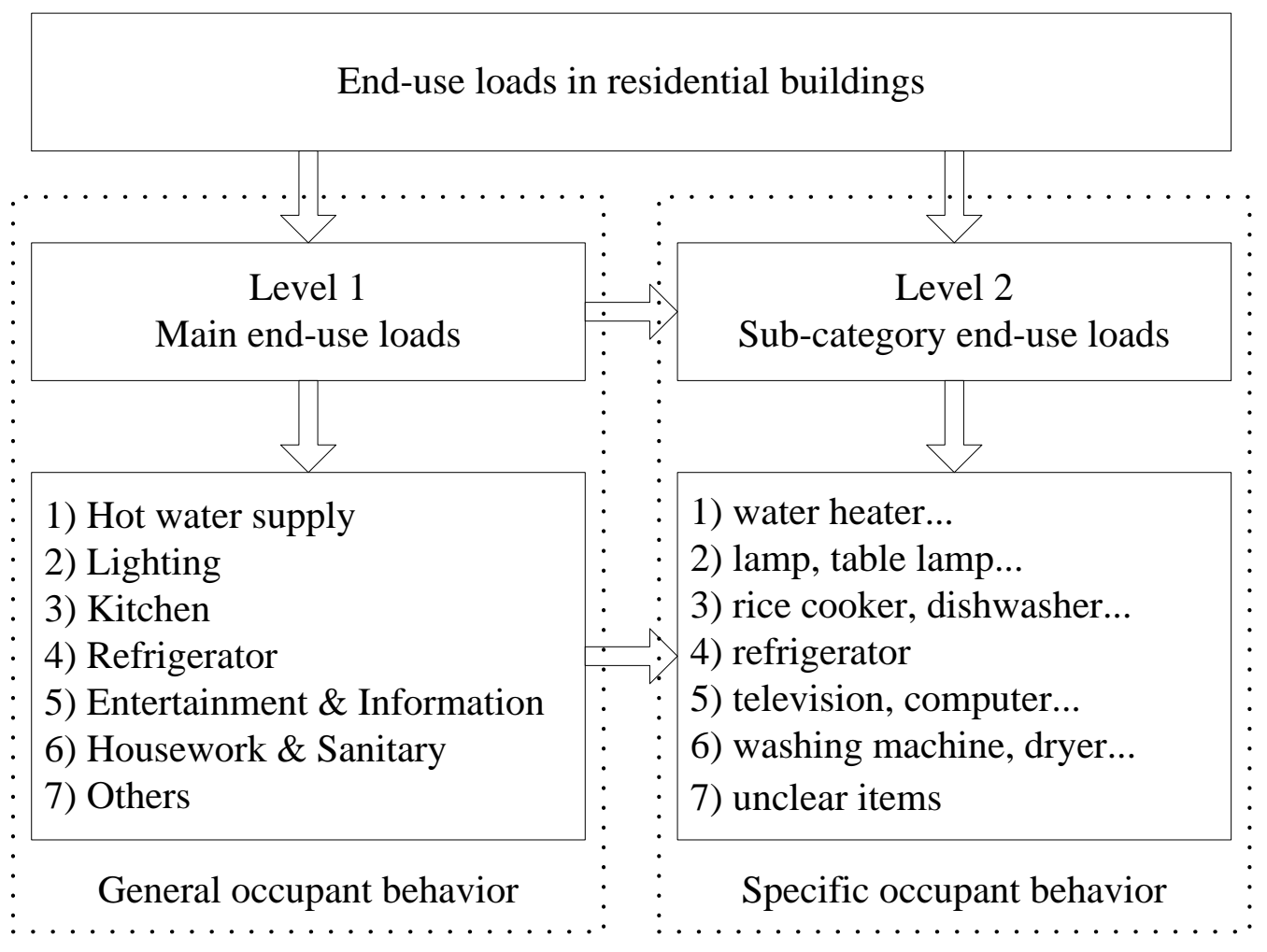

Fig.1. Two-level end-use loads

Level 1 loads are divided into seven main end-use loads), , each of which can be further divided into various end-users in level 2. The seven end-use loads in level 1 are assumed to be non-weather-dependent [10], due to the fact that the usage of these appliances (i.e. lighting, refrigerators, etc.) is mainly determined by occupants' presence and their behaviour, though it may also be partly impacted by weather conditions. At the same time, given that HVAC loads in the investigated buildings are primarily determined by weather conditions (especially outdoor air temperature), the HVAC load is not taken into consideration in this study though it may also partly be impacted by occupant behaviour. It should be mentioned that, the level 2 end-users are not fixed in different residential buildings since commonly different families have different household appliances. The level 1 and level 2 loads are mapped onto general occupant behavior, such as activities associating with lighting and hot water supply, and specific occupant behavior, such as the use of computers and washing machines. 
For demonstration purposes, a group of buildings is used to show the practical application of this methodology. Recommendations for improving occupant behaviour are provided for a selected building (case building) within this group.

The methodology is briefly described as follows.

(1) Identify energy-inefficient general occupant behavior in the case building.

(2) Identify a reference building for the case building to evaluate its energy-saving potential, and further determine its energy-inefficient general occupant behavior by comparison with the reference building.

(3) Identify energy-inefficient specific occupant behavior in the case building.

The proposed methodology can be demonstrated in a five-step process, as shown in Fig. 2.

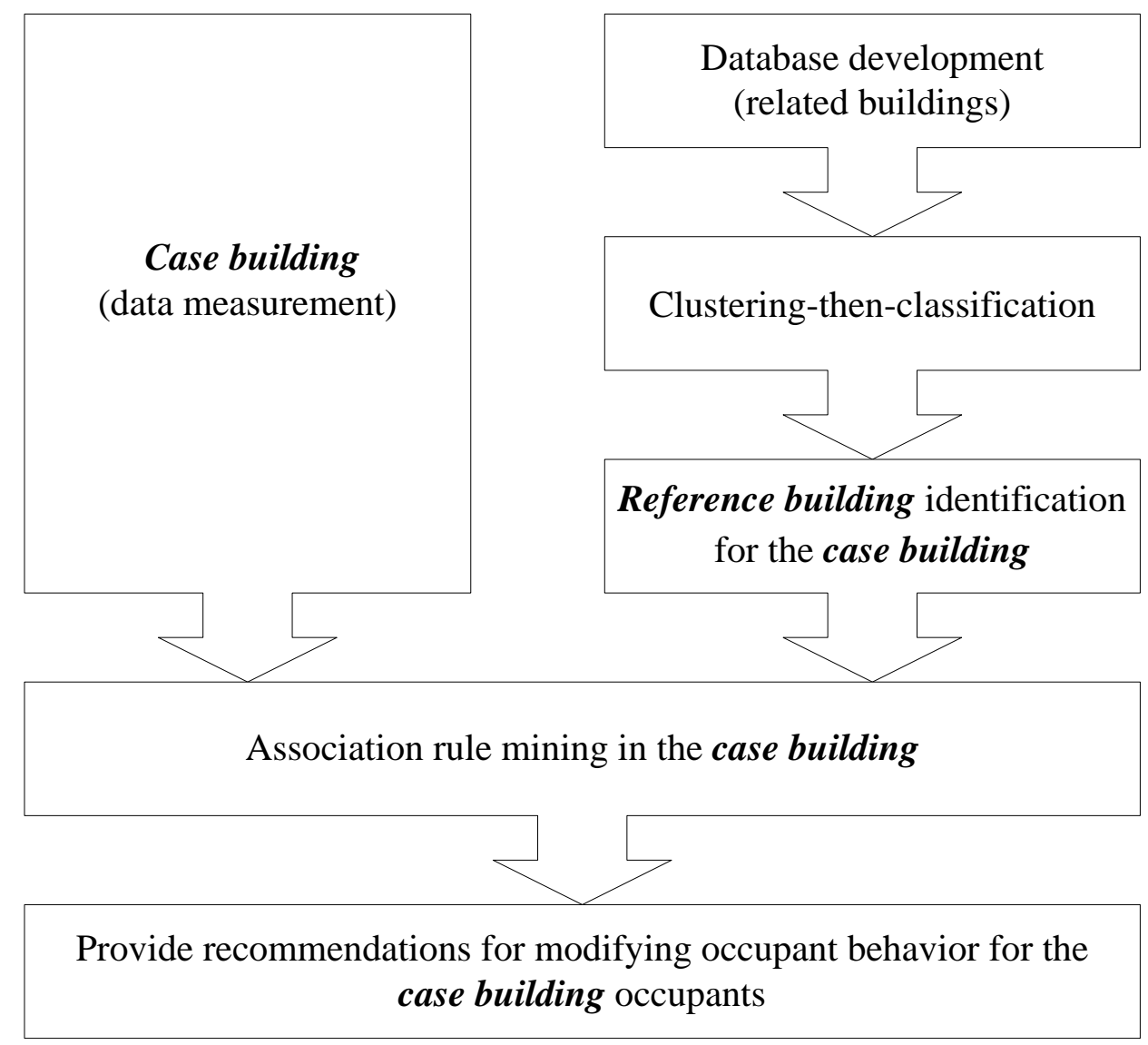

Fig.2. Methodology of evaluating and efficiently improving occupant behavior in the case building

Each step in this methodology is briefly explained as follows:

(1) First, a database should be developed based on the collection of measured data for the case building and other related buildings (e.g. buildings selected in the same city or country). The daily (or hourly) level 2 end-use loads should be measured, and the $\underline{\text { level } 1}$ end-use loads can be accumulated based on the level 2 data. The database 
should also contain information about building-related parameters, such as floor area and number of occupants.

(2) Through clustering analysis, all the related buildings in the database are clustered into different groups in terms of the level 1 loads (for each main end-use load, the annual per capita end-use loads is used for comparison). Accordingly, general occupant behavior in different buildings in the same group has a high similarity, but is quite different from that in other groups. Specifically, comparing with occupants in other clusters, on average each occupant in the same cluster consumes similar amounts of energy each year in terms of the seven level 1 end-use loads. Note that these seven loads are taken into consideration separately but simultaneously. Consequently, by comparing with other clusters, the characteristics of occupant behavior in each cluster can be identified. Such information can help building occupants to evaluate their own behavior among all the building owners in the database, thereby identifying general occupant behaviour which results in inefficient use of energy. Then, data classification based on the generated clusters is performed, and specifically, a decision tree [11] is developed. By using the generated decision tree, a building can be assigned to a specific cluster, provided its level 1 loads are available. In particular, once the case building has been assigned to a cluster, its general energy-inefficient occupant behaviour can be determined. It should be mentioned that, the decision tree was selected and used in this study due to the fact it can provide useful information which can help to understand the role of building occupant behavior in improving energy saving [12].

(3) Among the related buildings in the database, a reference building (RB) is identified for the case building to evaluate its energy-saving potential due to the occupant behavior modification. The RB is selected from the same cluster as the case building so that both of them have similar holistic occupant behavior patterns. The comparison with the RB also shows the case building occupants which general occupant behavior still need to be modified.

(4) After identifying the energy-inefficient general occupant behavior through clustering analysis and RB identification, it is necessary for the case building owner to know which specific activities and corresponding appliances deserve extra attention. Therefore, association rules are mined to identify the associations and correlations between various user activities in the case building, in order to highlight energy-saving opportunities.

(5) Recommendations for energy-efficient activities are provided for the case building occupants, so that they can modify their behavior.

In the following section, various data mining techniques employed in this methodology are first introduced. Then the steps in identifying a RB for the case building are explained. 


\subsection{Clustering-then-classification}

\subsubsection{Cluster analysis}

Cluster analysis is the process of grouping data objects into clusters so that objects in the same cluster have high similarity, while objects in different clusters have low similarity. Fig. 3 shows a clustering schema based on a hypothetical residential building data table. It contains various end-use loads such as supply hot water and lighting.

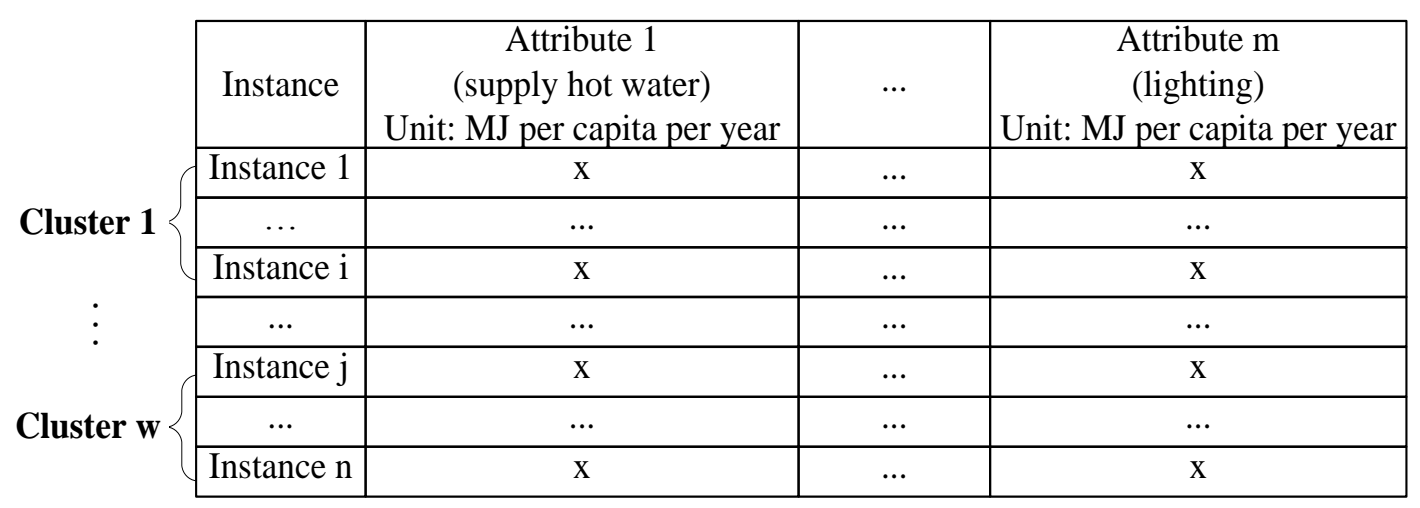

Fig.3. Clustering schema

This table consists of $m$ attributes and $n$ instances. Each attribute represents a variable and each instance denotes a building. All the instances are grouped into $w$ clusters. Accordingly, these $w$ clusters are homogeneous internally and heterogeneous between different clusters [11]. Such internal cohesion and external separation are based upon the various end-use loads, which can be mapped onto corresponding building occupant behavior. It implies that buildings in the same cluster have similar holistic occupant behavior patterns; while the patterns are significantly distinct for the buildings in different clusters.

The dissimilarity between data objects in the database is calculated using the distance between them in the cluster analysis. In this study, the most popular distance measure, Euclidean distance, was used [11]:

$$
d(k, l)=\sqrt{\left(x_{k 1}-x_{l 1}\right)^{2}+\left(x_{k 2}-x_{l 2}\right)^{2}+\cdots+\left(x_{k n}-x_{l n}\right)^{2}}
$$

where $k=\left(x_{k 1}, x_{k 2}, \ldots, x_{k n}\right)$ and $l=\left(x_{l 1}, x_{l 2}, \ldots, x_{l n}\right)$ are buildings. $x_{k 1}, \ldots, x_{k n}$ are $n$ parameters of $k$ and $x_{l l}, \ldots, x_{l n}$ are $n$ parameters of $l$.

Commonly used clustering algorithms include K-means, K-medoids, and CLARANS [11]. In this study, we employ the K-means, along with the open-source data mining program RapidMiner [13], to perform cluster analysis due to its efficiency and wide 
applicability.

The K-means algorithm is one of the simplest partition methods to solve clustering problems. Given a dataset $(D)$ containing $w$ objects, the K-means algorithm aims to partition these $w$ objects into $k$ clusters with two restraints: 1) the center of each cluster is the mean position of all objects in that cluster, 2) each object is assigned to the cluster with the closest center. The algorithm consists of five steps: 1) Randomly select $k$ observations from $D$ as the initial cluster centers, 2) Calculate the distance between each remaining observations and each initially chosen center, 3) Assign each remaining observation to the cluster with the closest center, 4) Recalculate the mean values, i.e., the cluster centers, of the new clusters, and 5) Repeat Steps 2 to 4 until the algorithm converges, meaning that the cluster centers do not change.

In RapidMiner, the performance of clustering algorithms is evaluated by using the Davies Bouldin index (DBI) [14]. This index is defined as the ratio of the sum of average distance inside clusters to distance between clusters.

$$
D B I=\frac{1}{n} \sum_{i=1}^{n} \max _{i \neq j}\left[\frac{R_{i}+R_{j}}{M_{i, j}}\right]
$$

where

$n$ : number of clusters,

$R_{i}, R_{j}$ : average distance inside cluster $i$ and cluster $j$ by averaging the distance between each cluster object and the cluster center,

$M_{i, j}$ : distance between cluster centers.

DBI is small if each cluster is comparatively dense; while different clusters are far from each other. Consequently, a smaller DBI indicates better performance of the clustering algorithm. It should be mentioned that the $\mathrm{K}$-means is quite sensitive to initial cluster centers. Therefore, different values should be tried so as to obtain the minimum DBI. At the same time, the number of clusters should be specified in advance.

\subsubsection{Classification analysis}

Among various classification algorithms, decision tree was selected and used in this study. The decision tree methodology is one of the most commonly used data mining methods $[11,15]$. It uses a flowchart-like tree structure to segregate a set of data into various predefined classes, thereby providing the description, categorization, and generalization of given datasets. As a logical model, decision tree shows how the value of a target variable can be predicted by using the values of a set of predictor variables. 


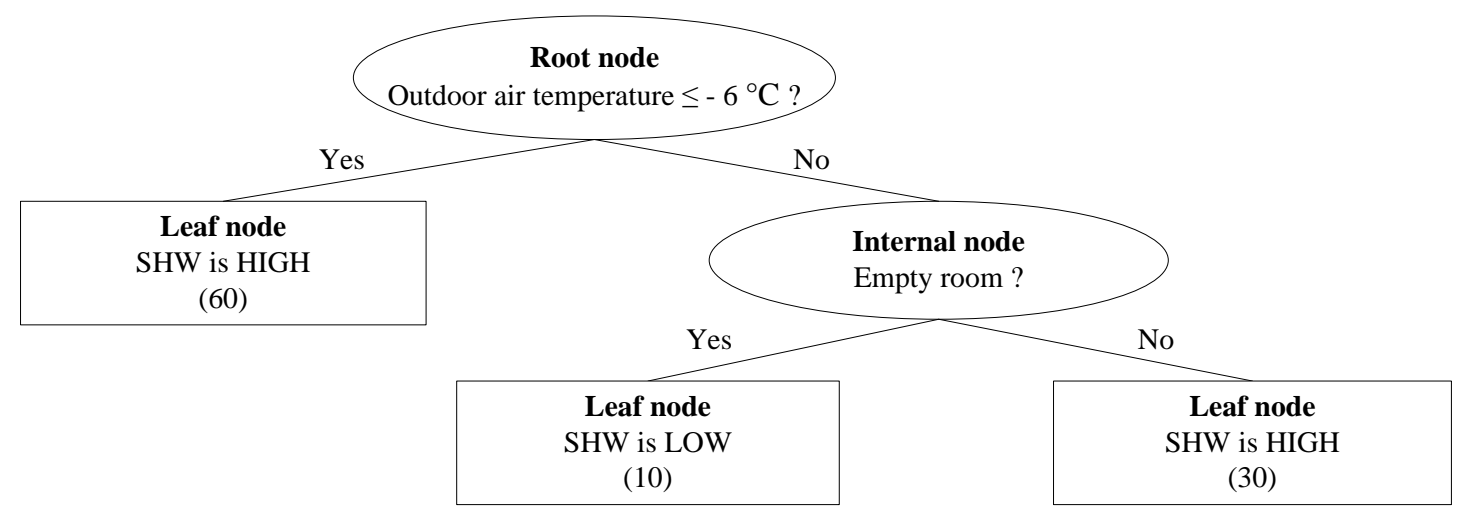

Fig.4. Schematic illustration of a simple hypothetical decision tree

Fig. 4 gives a simple decision tree indicating whether the supply hot water load (SHW) in a residential building is high or low in winter. For this example, assume 100 data records are used to build this decision tree, and that each record has three attributes: outdoor air temperature, occupant presence, and the level of SHW.

The target variable for the above decision tree is the level of SHW, with potential states being classified as either HIGH or LOW. The predictor variables are outdoor air temperature $\left(\leq-6^{\circ} \mathrm{C}\right.$ or $>-6^{\circ} \mathrm{C}$ ) and occupant presence (empty or occupied). As shown in Fig. 4, the decision tree consists of three kinds of nodes: root node, internal node, and leaf node. Root nodes and internal nodes denote a binary split test on an attribute while leaf nodes represent an outcome of the classification (i.e. a categorical target label). By using this decision tree, the SHW level classification ('i.e HIGH or LOW) can be predicted. For example, if the outdoor air temperature is higher than $6{ }^{\circ} \mathrm{C}$ and the room is empty, SHW is LOW; otherwise it is HIGH.

Decision tree generation is in general a two-step process, namely learning and classification, as shown in Fig. 5. In the learning process, the collected data is split into two subsets: a training set and a testing set. Creation of training sets and testing sets is an important part of evaluating data mining models. Usually, most of the data records in the database are arbitrarily selected for training and the remaining data records are used for testing. Note that training sets and testing sets should come from the same population but should be disjoint. Then, a decision tree generation algorithm takes the training data as an input, with the corresponding output being a decision tree. Commonly used decision tree generation algorithms include ID3 [15], classification and regression trees (CART) [16], and C4.5 [17]. In this study, we employ C4.5, along with the open-source data mining software RapidMiner [13], to build a decision tree. This software is selected due to its flexibility and wide applicability to different types of data. In the classification process, the accuracy of the obtained decision tree is first evaluated by making predictions against test data. The accuracy of a decision tree is measured by comparing the predicted target values with the true target values of the test data. If the accuracy is considered acceptable, the decision tree can be applied to new datasets for classification and prediction; otherwise, the reason for any 
inaccuracies should be identified and corresponding solutions should be adopted to address these problems.

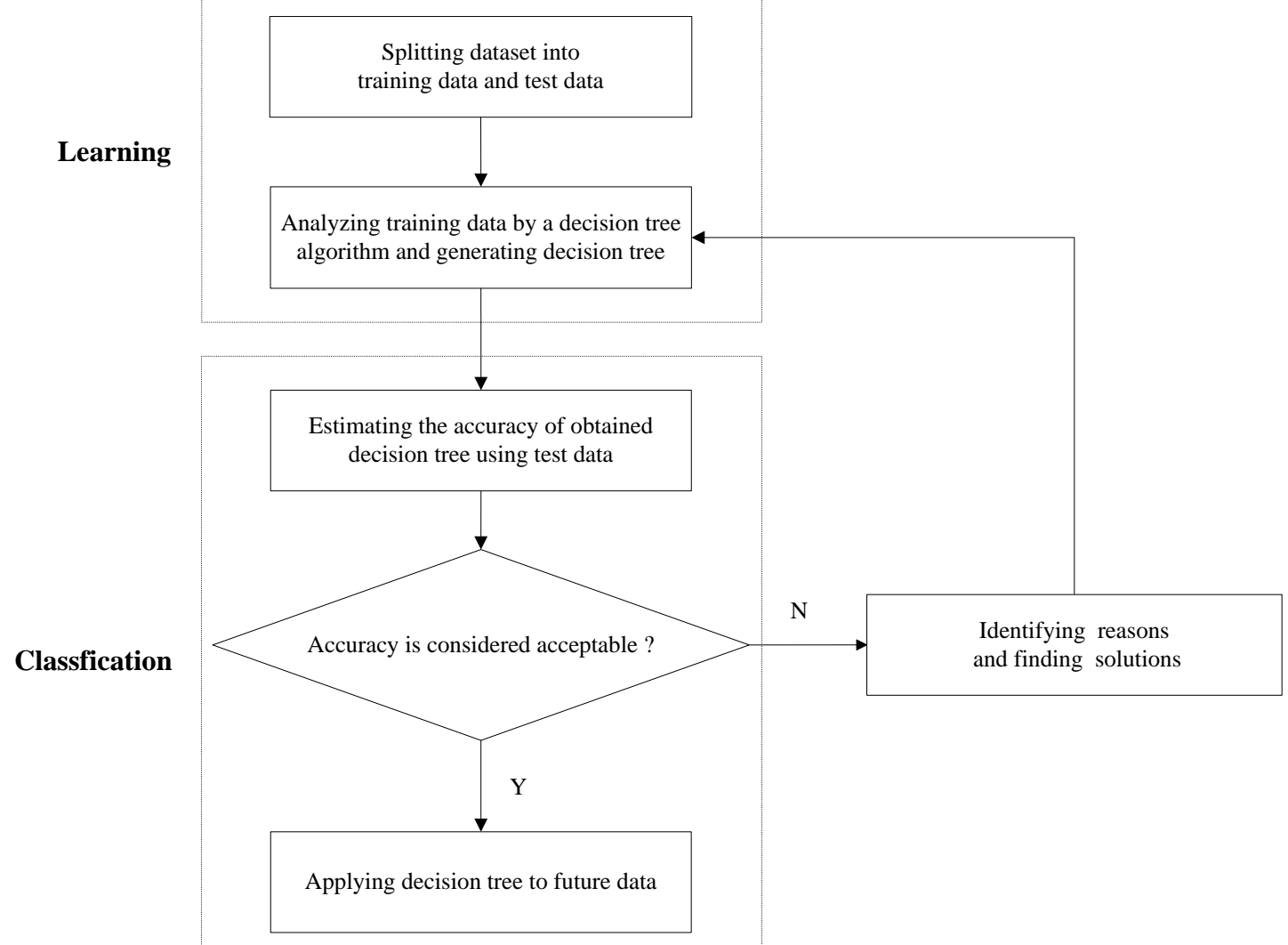

Fig.5. Procedure of decision tree generation

The procedure of generating a decision tree from the training data is as follows. Initially, all records in the training data are grouped together into a single partition. At each iteration, the algorithm chooses a predictor attribute that can "best" separate the target class values in the partition. The ability of a predictor attribute to separate the target class values is measured based on an attribute selection criterion, which was introduced in [12]. After a predictor attribute is chosen, the algorithm splits the partition into child partitions such that each child partition contains the same value of the chosen selected attribute. The decision tree algorithm iteratively splits a partition and stops when any one of the following terminating conditions is met:

- All records in a partition share the same target class value. Thus, the class label of the leaf node is the target class value.

- There are no remaining predictor attributes that can be used to further split a partition. In this case, the majority target class values become the label of the leaf node.

- There are no more records for a particular value of a predictor variable. In this case, a leaf node is created with the majority class value in the parent partition.

\subsubsection{Reference Building (RB) identification}

$\mathrm{RB}$ is normally utilized as a benchmark for comparison and the method of defining a 
RB depends on the purpose of study. In this study, the RB was defined to evaluate the energy-saving potential due to occupant behavior modification in the case building, and identify occupant behavior needing to be improved. Therefore, the definition of RB for the case building should comply with the following two rules:

Rule 1: The holistic occupant behavior patterns in RB and the case building should be as similar as possible. Different residential building occupants normally have different lifestyles and behavior patterns. In general, it is very difficult for building occupants to make dramatic lifestyle changes in order to reduce energy consumption. Hence, among the related buildings in the database, buildings with more similar occupant behavior patterns should be considered when evaluating the energy-saving potential for the case building. This implies that potential RB candidates should be chosen from buildings in the same cluster as the case building, since occupant behavior in the same cluster has a high similarity in comparison to one another, but is quite dissimilar to that in the other clusters.

Rule 2: Among all the potential RB candidates, the selected RB should have the highest similarity to the case building in terms of building-related parameters, such as outdoor temperature and floor area. This can also improve the reliability of comparative results between the two buildings. Euclidean distance can be used to define the similarity.

With consideration of the two rules, RB identification for the case building consists of the following steps:

Step 1: Assign the 'case building' to a cluster according to the level 1 loads and the generated decision tree;

Step 2: calculate the total energy consumption (i.e. the sum of the seven main end-use loads) in the case building and other buildings in the same cluster. Rank the total energy consumption in all these buildings;

Step 3: Identify the RB. Buildings in the same cluster with lower total energy consumption than the case building are used as potential RB candidates. Then, based on building-related parameters and Euclidean distance, the most similar building to the case building among the candidates can be found. This building is identified as $\mathrm{RB}$ for the case building.

\subsubsection{Association rule mining}

In data mining, association rules are often used to represent patterns of parameters that are frequently associated together. An example is given to illustrate the concept of association rules. Assume that 100 occupants live in 100 different rooms in the same building and each room has both a window and a door. Moreover, 40 occupants open the windows and 20 occupants open the doors. If 10 occupants open both the windows and doors simultaneously, it can be calculated that these 10 occupants account for $10 \%$ of all the building occupants $(10 / 100=10 \%)$, and $25 \%$ of the 
occupants who open windows $(10 / 40=25 \%)$. Then, the information that occupants who open windows also tend to open doors at the same time can be represented in the following association rule:

open_windows $\rightarrow$ open_doors [support $=10 \%$, confidence $=25 \%$ ]

In this statement, support and confidence are employed to indicate the validity and certainty of this association rule. Different users or domain experts can set different thresholds for support and confidence according to their own requirements, in order to discover useful knowledge eventually. Accordingly, the association rule mining (ARM) can be defined as finding out association rules that satisfy the predefined minimum support and confidence from a given database.

Mathematically, support and confidence can be calculated by probability, $\mathrm{P}(\mathrm{X} \cup \mathrm{Y})$, and conditional probability, $\mathrm{P}(\mathrm{Y} \mid \mathrm{X}$ ), respectively ( $\mathrm{X}$ denotes the premise and $\mathrm{Y}$ denotes the consequence in the sequence). That is,

$$
\begin{aligned}
& \operatorname{support}(\mathrm{X} \rightarrow \mathrm{Y})=\mathrm{P}(\mathrm{X} \cup \mathrm{Y}) \\
& \operatorname{confidence}(\mathrm{X} \rightarrow \mathrm{Y})=\mathrm{P}(\mathrm{Y} \mid \mathrm{X})
\end{aligned}
$$

Another concept, lift, which is similar to confidence, is commonly used to demonstrate the correlation between the occurrence of $\mathrm{X}$ and $\mathrm{Y}$ when conducting the ARM. Mathematically,

$$
\operatorname{lift}(\mathrm{X} \rightarrow \mathrm{Y})=\frac{\mathrm{P}(\mathrm{X} \cup \mathrm{Y})}{\mathrm{P}(\mathrm{X}) \mathrm{P}(\mathrm{Y})}=\frac{\mathrm{P}(\mathrm{Y} \mid \mathrm{X})}{\mathrm{P}(\mathrm{Y})}
$$

Particularly, a lift value greater than 1 represents a positive correlation (the higher this value is, the more likely that $\mathrm{X}$ coexists with $\mathrm{Y}$, and there is a certain relationship between $X$ and $Y$ [18]) while a lift value less than 1 represents a negative correlation. If the value is equal to 1 , i.e. $\mathrm{P}(\mathrm{X} \cup \mathrm{Y})=\mathrm{P}(\mathrm{X}) \mathrm{P}(\mathrm{Y})$, the occurrence of $\mathrm{X}$ is independent of the occurrence of $\mathrm{Y}$, and there is no correlation between $\mathrm{X}$ and $\mathrm{Y}$.

Commonly used ARM algorithms include the Apriori algorithm and the frequent-pattern growth (FP-growth) algorithm [11]. In this study, we employ the FP-growth algorithm, along with the open-source data mining software RapidMiner [13], to mine association rules due to its high efficiency and wide applicability. For the specific algorithm of FP-growth the reader can refer to [11].

Additionally, in order to perform the ARM, the value of quantitative attributes generally needs to be classified into categorical values. Considering that most attributes used in the ARM in this study are end-use electricity loads, a two-interval scale (i.e., HIGH and LOW) was applied to represent high and low energy 
consumption. Such high and low energy consumption can then be qualitatively mapped onto energy-inefficient and energy-efficient occupant behavior. It should be mentioned that HIGH and LOW quite possibly, but do not necessarily, correspond to energy-inefficient and energy-efficient occupant behaviour in practice. For example, less energy efficient appliances will also cause higher energy consumption. However, given that energy-inefficient behaviour will waste energy and normally cause high energy consumption, such mapping was still used in this study. Consequently, the results need to be carefully analyzed and energy-inefficient behaviour should be eventually identified based on practical occupant behaviour patterns. Specifically, for each quantitative attribute, data ranged from the average of the maximum and minimum to the maximum value is 'HIGH', and data ranged from the minimum value to the average of the maximum and minimum is ' $\mathrm{LOW}$ '.

\section{Data collection and pre-processing}

\subsection{Data collection}

To evaluate and improve the energy performance of residential buildings, a project entitled "Investigation on Energy Consumption of Residents All over Japan" was carried out by the Architecture Institute of Japan from December 2002 to November 2004 [19]. For this project, field surveys on energy-related data and other relevant information were carried out in 80 residential buildings located in six different districts in Japan: Hokkaido, Tohoku, Hokuriku, Kanto, Kansai, and Kyushu. Table 1 shows the survey items and corresponding investigation methods. Fig. 6 shows the measuring instruments which were used to monitor temperature and consumptions of electricity, gas, and/or kerosene. As mentioned previously, the collected data can be divided into two levels. However, for the level 2 data, currently only daily data is available (instead of data at 1 or 5 minute time steps).

\section{Table 1}

Investigation items and methods

\begin{tabular}{cccc}
\hline Method & \multicolumn{2}{c}{ Survey items } & Measuring time \\
\hline \multirow{2}{*}{$\begin{array}{c}\text { Field } \\
\text { measurement }\end{array}$} & $\begin{array}{c}\text { Different end-use loads of all } \\
\text { kinds of fuel }\end{array}$ & $\begin{array}{c}\text { Electricity } \\
\text { Gas }\end{array}$ & $\begin{array}{c}\text { Measured every minute } \\
\text { Measured every 5 minutes } \\
\text { Questionnaire } \\
\text { survey }\end{array}$ \\
\cline { 2 - 4 } & Lerosene & Measured every 5 minutes \\
\hline Inquiring survey & Other issues, such as basic building information & Measured every 15 minutes \\
\hline
\end{tabular}



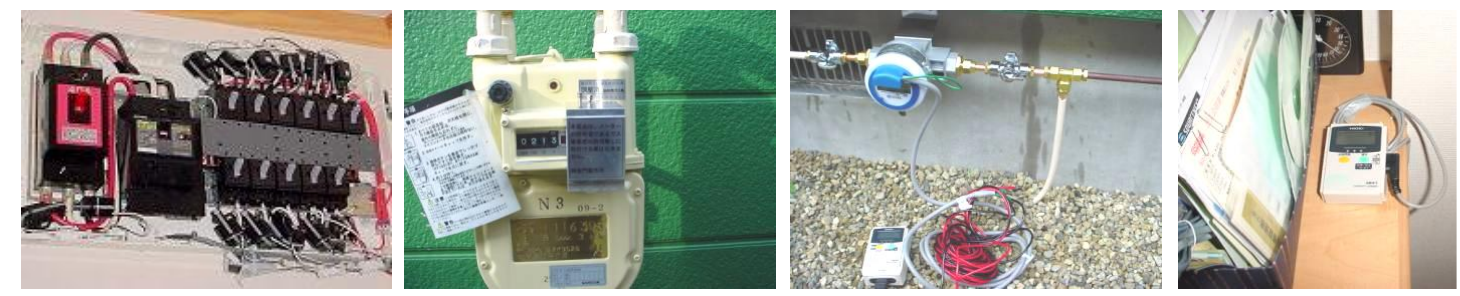

Fig.6. Measuring instruments (from left to right: electricity, gas, kerosene and air temperature)

\subsection{Data pre-processing}

\subsubsection{Data integration and reduction}

Scrutinizing the data from the 80 buildings, it was found that only 67 sets were complete, while 13 sets had missing values of energy consumption data. Data integration was carried out for the detection and resolution of data value conflicts. For example, diverse energy units of different kinds of primary energy sources used by the various buildings (including electricity, natural gas, and kerosene) were converted to MJ based on conversion coefficients in Table 2. After conversion, they could be added directly. Then, data reduction was performed to obtain a smaller representation of the original data. For example, readings of each main end-use load at different intervals (e.g., 1 or 5 minutes) were averaged over one year. The resulting data was stored in a database.

\section{Table 2}

Conversion coefficients of different fuels

\begin{tabular}{ccc}
\hline Fuel & Conversion coefficient & Unit \\
\hline Electricity & 3.6 & $\mathrm{MJ} / \mathrm{kWh}$ \\
City gas (4A-7C) & 20.4 & $\mathrm{MJ} / \mathrm{Nm}^{3}$ \\
City gas (12A-13C) & 45.9 & $\mathrm{MJ} / \mathrm{Nm}^{3}$ \\
Liquefied petroleum gas (LPG) & 50.2 & $\mathrm{MJ} / \mathrm{Nm}^{3}$ \\
Kerosene & 36.7 & $\mathrm{MJ} / \mathrm{L}$ \\
\hline
\end{tabular}

\subsubsection{Case building selection}

As mentioned earlier, for demonstration purposes, one building with the most comprehensive household appliances should be selected as the case building, and the remaining 66 buildings are used for both clustering-then-classification and RB identification. Data inspection indicates that a building located in Hokkaido has the most appliances, as shown in Table 3. Table 3 also shows some measured environmental parameters of this building such as indoor air temperature and humidity. These parameters will also be used in the ARM to analyze the associations between them and occupant behaviour.

\section{Table 3}

Appliances in the case building and environmental parameters used in ARM

\begin{tabular}{lllll}
\hline No. & Appliances/ & No. & Appliances/ & No.
\end{tabular}




\begin{tabular}{cccccc}
\hline & indoor parameters & & indoor parameters & indoor parameters \\
\hline 1 & Heating boiler & 16 & TV (other rooms) & 31 & Living room temperature \\
2 & Hot water boiler & 17 & TV (standby power) & 32 & Living room humidity \\
3 & Kerosene heater & 18 & Video & 33 & Bedroom (1F) temperature \\
4 & Ventilator & 19 & Phone & 34 & Master bedroom (2F) temperature \\
5 & Air cleaner & 20 & Telephone handset & 35 & Total energy consumption \\
6 & Lamp $\left(1 \mathrm{~F}^{\mathrm{a}^{*}}\right)$ & 21 & Iron & 36 & SHW \\
7 & Lamp $\left(2 \mathrm{~F}^{\mathrm{b}^{*}}\right)$ & 22 & Vacuum cleaner & 37 & LIGHT \\
8 & Table lamp & 23 & Washing machine (1F) & 38 & KITCH \\
9 & IH heater & 24 & Washing machine (2F) & 39 & REFRI \\
10 & Dishwashers & 25 & Living room outlet & 40 & E\&I \\
11 & Microwave, toaster, coffee & 26 & Rest room outlet (1F) & 41 & H\&S \\
12 & Bidet & 27 & Rest room outlet (2F) & 42 & OTHER \\
13 & Boom box & 28 & Outdoor air temperature & & \\
14 & TV (Dining room) & 29 & Outdoor relative humidity & & \\
15 & TV (master bedroom $2 \mathrm{~F})$ & 30 & Outdoor air velocity & & \\
\hline $\mathrm{a}^{*}$ first floor, ${ }^{\mathrm{b}^{*}}$ second floor. & & &
\end{tabular}

Table 4 shows the statistical data of the level 1 loads for the remaining 66 buildings. Clearly, it can be seen that each main end-use load is spread over a wide range, which implies fairly large energy-saving potential by improving occupant behavior.

\section{Table 4}

Statistical data of the seven main end-use loads for the 66 buildings (unit: MJ per capita per year)

\begin{tabular}{ccccc}
\hline End-use load & Min & Max & Average & Standard deviation \\
\hline SHW & 994.945 & 11649.175 & 4695.497 & 2616.451 \\
LIGHT & 130.372 & 2938.521 & 1311.695 & 846.283 \\
KITCH & 110.761 & 5321.785 & 971.773 & 786.056 \\
REFRI & 390.136 & 2667.98 & 883.033 & 439.375 \\
E\&I & 106.254 & 2301.679 & 727.136 & 480.946 \\
H\&S & 64.137 & 2102.968 & 400.303 & 385.46 \\
OTHER & 55.259 & 2374.798 & 738.422 & 564.375 \\
\hline
\end{tabular}

\subsubsection{Data transformation for cluster analysis}

Before performing the cluster analysis on the level 1 data, it should be noted that the loads, which were mapped onto various corresponding user activities, have different ranges. Moreover, the activities were considered to be of equal importance in this study. In order to prevent the loads with large ranges from outweighing those with comparatively smaller ranges, min-max normalization was applied before clustering the buildings in terms of the seven main end-use loads. Specifically, the min-max normalization [11] can scale the values so that they fall within a predetermined range. The main advantage of the min-max normalization lies in its ability to reserve the 
relationships between the initial data, since it carries out a linear normalization. Assume that $x_{\max }$ and $x_{\min }$ are the original maximum and minimum values of a numerical attribute (i.e. the level_1 end-use loads in this study). By using the min-max normalization, a value of this attribute (e.g. $x$ ) can be transformed to $x^{\prime}$ in the new specified range $\left[x_{\text {min }}^{\prime}, x^{\prime}{ }_{\text {max }}\right]$ by calculating

$$
x^{\prime}=\frac{x-x_{\text {min }}}{x_{\text {max }}-x_{\text {min }}}\left(x^{\prime}{ }_{\text {max }}-x^{\prime}{ }_{\text {min }}\right)+x_{\text {min }}^{\prime}
$$

In this study, the new range is defined as $[0,1]$. Table 5 shows the statistical data of the level 1 loads for the remaining 66 buildings after min-max normalization.

Table 5

Statistical data after normalization

\begin{tabular}{ccccc}
\hline End-use load & Min & Max & Average & Standard deviation \\
\hline SHW & 0 & 1 & 0.347 & 0.246 \\
LIGHT & 0 & 1 & 0.421 & 0.301 \\
KITCH & 0 & 1 & 0.165 & 0.151 \\
REFRI & 0 & 1 & 0.216 & 0.193 \\
E\&I & 0 & 1 & 0.283 & 0.219 \\
H\&S & 0 & 1 & 0.165 & 0.189 \\
OTHER & 0 & 1 & 0.295 & 0.243 \\
\hline
\end{tabular}

\subsubsection{Removal of outliers for conducting ARM in the case building}

Outliers are data objects whose values are grossly different (i.e. much higher or lower) from others in the database. Outliers regularly occur in building energy consumption measurement. They are often indicative of measurement errors, and thus must be removed. Removal of outliers plays a crucial role in preparing for the ARM, since outliers produce a large measure of skewness and have a significant influence on the partition of attribute values into different intervals. For example, suppose an attribute ranges from 0 to 10 , and can be discretized into two intervals, $[0,5)$ and $[5$, 10] (or LOW and HIGH), by using the methods mentioned previously. If there exists an outlier (e.g. 30), then the two intervals are $[0,15)$ and $[15,30]$ (or LOW and HIGH) by using the same method. Accordingly, all the data are defined as LOW except the outlier, which is not actually true.

Various methods can be used for effective detection and removal of outliers. In this study, a method based on the lower quartile $\left(\mathrm{Q}_{1}\right)$ and the upper quartile $\left(\mathrm{Q}_{3}\right)$ of the standard boxplot was used due to its simplicity [20]. Specifically, outlying values can be distinguished using the following two rules:

Rule 1: data values that are less than $\mathrm{Q}_{1}-1.5 \times\left(\mathrm{Q}_{3}-\mathrm{Q}_{1}\right)$ are defined as outliers Rule 2: data values that are larger than $\mathrm{Q}_{3}+1.5 \times\left(\mathrm{Q}_{3}-\mathrm{Q}_{1}\right)$ are defined as outliers

With consideration of the seasonality of occupant behavior, the ARM was performed 
based on seasonal data instead of annual data in this study for demonstration purposes. Given that the case building is located in Hokkaido, the coldest area in Japan, the winter data in 2003 was mined to generate association rules. Fig. 7 shows the distribution of two intervals of all the ARM attributes after the removal of outliers. Note that the numbers in the abscissa represent the ARM attributes, and correspond to the number in Table 3. Clearly, it can be observed that most of the percentages range from $30 \%$ to $70 \%$, indicating a roughly uniform distribution.

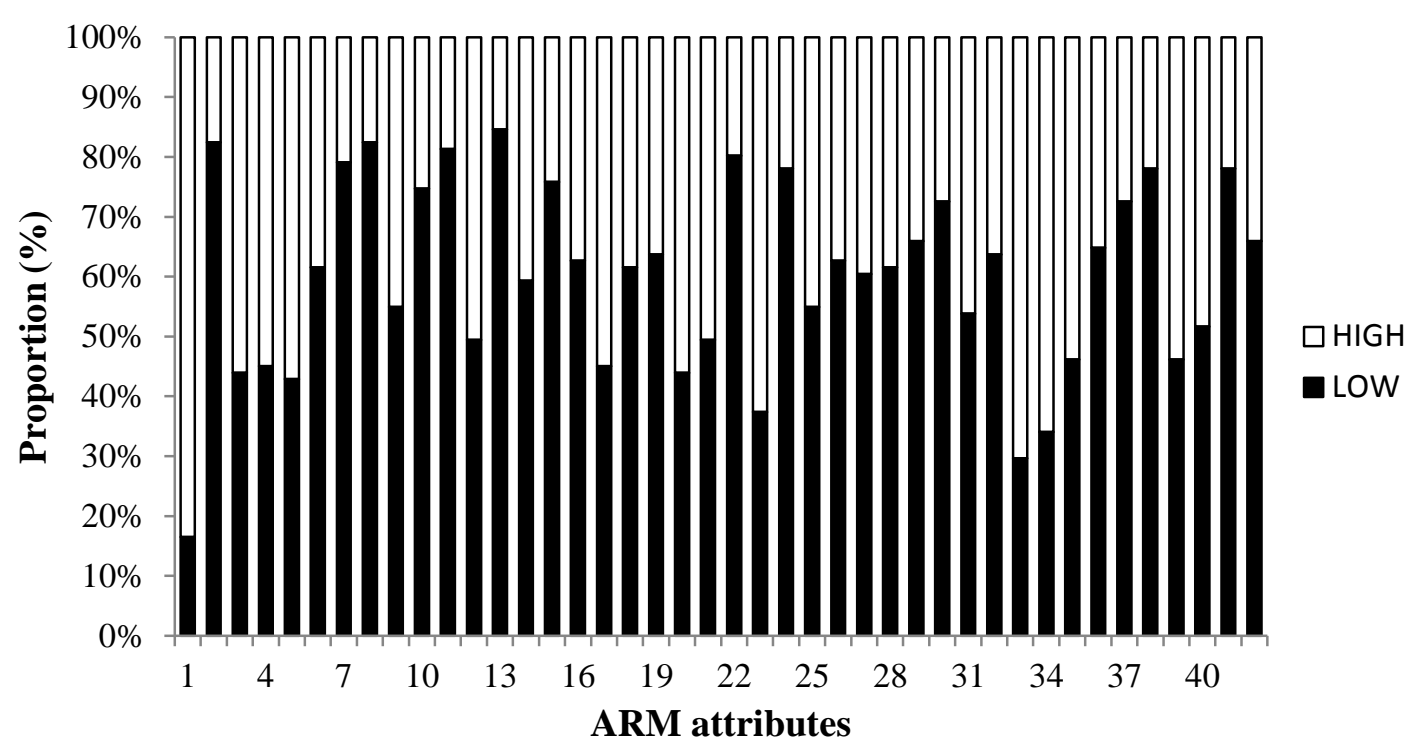

Fig.7. Distribution of two intervals of all ARM attributes after the removal of outliers

\section{Results and discussion}

\subsection{Clustering-then-classification}

\subsubsection{Clustering results}

After data pre-processing, the cluster analysis was conducted for the 66 buildings using the RapidMiner. With consideration of the size of the database, four clusters were determined by the K-means algorithm and the performance vector (Davies Bouldin index, DBI). The results of the cluster analysis are given in Table 6. Cluster centroid, which represent the mean value for each dimension, were used to characterize building occupant behavior in the four clusters. For example, in comparison with building occupant behavior in the other clusters, user activities in cluster_2 caused medium energy consumption in supply hot water (the cluster centroid of SHW in this cluster is 0.440 , which is of medium value among the four clusters), high energy consumption in lighting, medium energy consumption in kitchen, etc. Moreover, cluster_2 has significantly higher energy consumption for lighting; this indicates that, in general, building owners in cluster_2 should give primary consideration to the activities related to lighting in order to save energy. Similarly, other clusters can be explained. It should be noted that nearly half of the data records (44\%) were grouped into cluster_1, which represents low energy consumption in most of the main end-use loads. A possible explanation for this is that 
a good portion of Japanese families have a high degree of awareness regarding energy-savings. In addition, among the seven attributes and four clusters, H\&S has the largest maximum/minimum ratio $(0.509 / 0.088=6.5)$, while $\mathrm{KITCH}$ has the lowest maximum/minimum ratio $(0.268 / 0.144=1.91)$. This indicates that occupant behavior related to H\&S differs significantly between the four clusters; and deserves extra attention in occupant behavior improvement; on the contrary, the total energy consumption caused by KITCH-related user activities has a narrow gap between different clusters, which implies relatively small energy-saving potential for modifying such kind of activities.

\section{Table 6}

Centroid of each cluster and statistics on the number and percentage of instances assigned to different clusters

\begin{tabular}{ccccc}
\hline Attribute & Cluster_1 & Cluster_2 & Cluster_3 & Cluster_4 \\
\hline SHW & 0.266 & 0.440 & 0.738 & 0.215 \\
LIGHT & 0.262 & 0.881 & 0.291 & 0.288 \\
KITCH & 0.144 & 0.181 & 0.268 & 0.140 \\
REFRI & 0.119 & 0.255 & 0.372 & 0.296 \\
E\&I & 0.218 & 0.169 & 0.572 & 0.403 \\
H\&S & 0.088 & 0.167 & 0.509 & 0.150 \\
OTHER & 0.136 & 0.430 & 0.231 & 0.500 \\
Clustered buildings and proportion & $29(44 \%)$ & $16(24 \%)$ & $7(11 \%)$ & $14(21 \%)$ \\
\hline
\end{tabular}

Table 7 shows the number of buildings in various districts in each cluster. Clearly, the distribution of buildings in various districts is roughly even, especially in cluster_1 and cluster_4. Such a distribution indicates that the attributes in the cluster analysis are not dependent on weather (otherwise buildings in the same districts would tend to be grouped together), which is consistent with the assumption that the seven main end-use loads in clustering analysis are non-weather-dependent components.

Table 7

The number of buildings in various districts in each cluster

\begin{tabular}{ccccccc}
\hline cluster & Hokkaido & Tohoku & Hokuriku & Kanto & Kansai & Kyusyu \\
\hline cluster_1 & 6 & 3 & 7 & 3 & 5 & 5 \\
cluster_2 & 0 & 4 & 0 & 8 & 2 & 2 \\
cluster_3 & 1 & 2 & 4 & 0 & 0 & 0 \\
cluster_4 & 3 & 2 & 1 & 1 & 5 & 2 \\
\hline
\end{tabular}

\subsubsection{Classification by decision tree}

\subsubsection{Generation of decision tree}

After the four clusters were generated, a decision tree was constructed to assign buildings to a specific cluster provided their main end-use loads are available, as shown in Fig.8. C4.5 algorithm was used in RapidMiner to build the decision tree. 


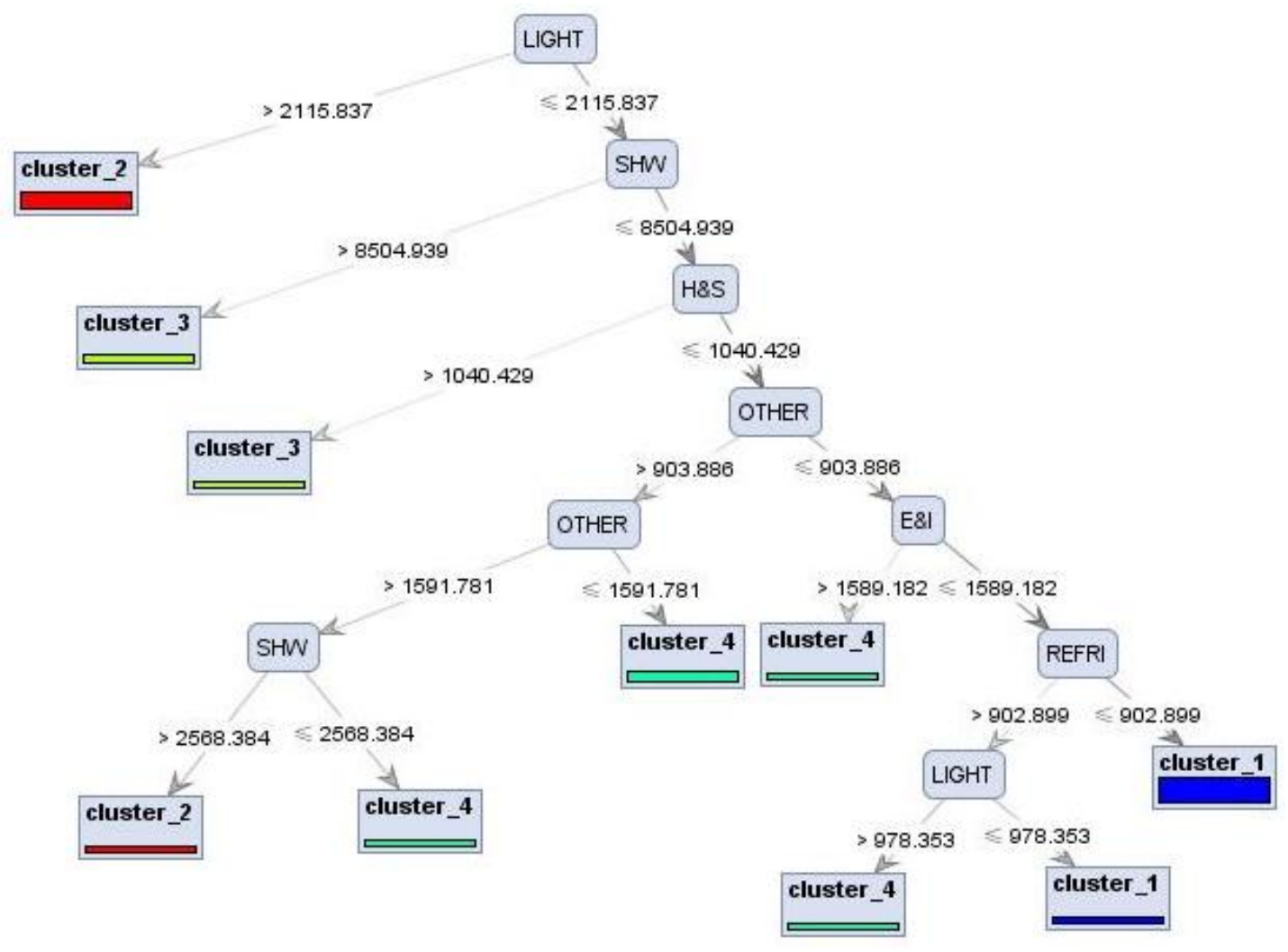

Fig.8. Decision tree for the prediction of cluster attribution

The decision tree includes a total of 19 nodes among which 10 are leaf nodes. The colors in the leaf nodes indicate the purity of classification in the nodes. A pure color in a node implies that all the records in this node are correctly classified. Clearly, all the data records in the training dataset are correctly classified in this decision tree.

\subsubsection{Evaluation of the decision tree}

In order to evaluate the accuracy of the generated decision trees, the RapidMiner analysis report also provides a confusion matrix for data analysts. In this study, a four-dimensional confusion matrix was built since the decision tree has four target variables, as shown in Table 8. 


\section{Table 8}

Confusion matrix

\begin{tabular}{cccccc}
\hline & & \multicolumn{5}{c}{ Predicted data records } \\
\cline { 3 - 6 } & & Cluster_1 & Cluster_2 & Cluster_3 & Cluster_4 \\
\hline \multirow{2}{*}{ Actual } & Cluster_1 & 7 & 0 & 0 & 0 \\
\multirow{2}{*}{ data } & Cluster_2 & 1 & 4 & 0 & 0 \\
records & Cluster_3 & 1 & 0 & 1 & 0 \\
& Cluster_4 & 2 & 0 & 0 & 4 \\
\hline
\end{tabular}

In this table, the rows indicate the number of actual data records used for testing in each cluster; and the columns represent the number of predicted data records generated by applying the decision tree to the actual data records. For example, the first column shows that 7 records in cluster_1 were correctly classified; while one record in cluster_2, one record in cluster_3, and two records in cluster_4 were misclassified into cluster_1. Therefore, the accuracy of this decision tree, which is also called 'recall' in the data mining domain, can be calculated as $(7+4+1+4) \div(7+4+1+4+1+1+2)=80 \%$, which is basically acceptable though relatively low. This may be partly ascribed to the small size of database. Moreover, data records in cluster_2, cluster_3, and cluster_4 are misclassified into cluster_1 (at least one record in each cluster and four records totally), while data records in cluster_1 are not misclassified into the other clusters. Such information indicates that cluster_1 is more prone to be misclassified than the other clusters. This may have occurred since nearly half of the data records in the database are in cluster_1, which makes the decision tree more sensitive to this cluster. An even distribution among the four clusters in the database would possibly improve the accuracy. In addition, the sum of values in the matrix corresponds to the number of data records used for model testing. Clearly 20 records in the database were randomly selected by RapidMiner for testing, which also implies that 46 data records were used to establish the decision tree.

\subsubsection{Utilization of the decision tree}

The decision tree can be utilized to predict the cluster attribution of new buildings according to the main end-use loads. Such predictions can be easily made by traversing a path from the root node to a leaf node. Take the node in the lower left corner in Fig. 8 as an example. The prediction can be made as follows: for a building, if LIGHT $\leqslant 2115.837$ and SHW $\leqslant 8504.939$ and H\&S $\leqslant 1040.429$ and OTHER > 903.886 and OTHER > 1591.781 and SHW > 2568.384, then this building belongs to cluster_2.

Besides the prediction of cluster attribution, useful information can also be extracted from the decision tree so as to help understand building occupant behavior improvement. For example, various attributes are selected by the decision tree 
algorithm to split the nodes; and their degrees of closeness to the root node determines the number of records impacted. Therefore, the closer an attribute is to the root node, the more significant it affects the cluster attribution. Clearly the attribute significance in the decision tree can be ranked as: LIGHT > SHW > H\&S > OTHER > E\&I > REFRI. Such information indicates a general descending order of occupant behavior deserving attention when modifying user activities in Japanese residential buildings. Moreover, among the seven end-use loads, KITCH does not appear in the decision tree. This may have occurred due to the narrow gap between energy consumption caused by KITCH-related occupant behavior among the four clusters (see section 4.1.1), and thus KITCH has the weakest influence on the cluster attribution.

\section{2. $R B$ identification}

In order to demonstrate the methodology, a case building with the most comprehensive household appliances was selected for case study. Table 9 shows the level 1 loads in this case building.

\section{Table 9}

End-use data in the case building (unit: MJ per capita per year)

\begin{tabular}{cccccccc} 
SHW & LIGHT & KITCH & REFRI & E\&I & H\&S & OTHER & Sum \\
3882.699 & 582.052 & 250.600 & 1541.394 & 1799.530 & 621.743 & 336.592 & 9014.610 \\
\hline
\end{tabular}

Based on the decision tree, the cluster attribution of the case building can be predicted as follows:

Step 1: Examine the value of LIGHT, i.e., the attribute in the root node. Since LIGHT $=582.052$, the node test in the right branch LIGHT $\leqslant 2115.837$ is satisfied, then go to the right-side child node;

Step 2: Examine the value of SHW. Since SHW $=3882.699$, the node test in the right branch $S H W \leqslant 8504.939$ is satisfied, then go to the right-side child node;

Step 3: Examine the value of $H \& S$. Since $H \& S=621.743$, the node test in the right branch $H \& S \leqslant 1040.429$ is satisfied, then go to the right-side child node;

Step 4: Examine the value of OTHER. Since OTHER $=336.592$, the node test in the right branch $O T H E R \leqslant 903.886$ is satisfied, then go to the right-side child node;

Step 5: Examine the value of E\&I. Since E\&I = 1799.530, the node test in the left branch $E \& I \leqslant 1589.182$ is satisfied, then go to the left-side child node, which is a leaf node. As a result, the decision tree in Fig. 8 predicts that the case building belongs to cluster_4.

Comparing with the other three clusters, cluster_4, as shown in Table 6, can be characterized as the building group with high energy consumption in OTHER, medium high energy consumption in REFRI and E\&I. Therefore, the case building occupants should manage to improve their behavior related to OTHER, REFRI, and E\&I. 
After the prediction of cluster attribution, the sum of the seven main end-use loads in the buildings in cluster_4 was calculated and ranked. Table 10 shows these loads and their sum in the 14 buildings in cluster_ 4 in ascending order.

\section{Table 10}

The main end-use loads in the 14 buildings in cluster_4 (Unit: MJ per capita per year)

\begin{tabular}{ccccccccc}
\hline No. & SHW & LIGHT & KITCH & REFRI & E\&I & H\&S & OTHER & Sum \\
\hline 1 & 1691.656 & 744.428 & 1141.730 & 898.208 & 468.707 & 83.617 & 1670.297 & 6698.644 \\
2 & 2757.408 & 981.880 & 662.657 & 645.977 & 388.737 & 317.828 & 1100.376 & 6854.487 \\
3 & 1464.821 & 287.523 & 936.880 & 924.793 & 1958.911 & 504.171 & 845.352 & 6922.450 \\
4 & 2471.123 & 865.524 & 1065.978 & 879.398 & 608.810 & 162.782 & 942.645 & 6996.259 \\
5 & 1782.779 & 1099.852 & 322.597 & 1773.017 & 2092.484 & 142.018 & 556.186 & 7768.933 \\
6 & 3337.796 & 558.252 & 411.807 & 1013.407 & 1060.430 & 360.339 & 1253.659 & 7995.690 \\
7 & 3123.892 & 1094.065 & 1418.592 & 1055.741 & 803.612 & 160.549 & 1288.371 & 8944.821 \\
8 & 2694.449 & 1758.554 & 621.970 & 1170.580 & 1109.116 & 503.125 & 1220.652 & 9078.446 \\
9 & 3348.343 & 1407.656 & 1474.419 & 1046.065 & 768.032 & 550.396 & 739.591 & 9334.501 \\
10 & 5224.677 & 617.440 & 724.771 & 565.889 & 498.162 & 186.758 & 1530.789 & 9348.487 \\
11 & 4801.992 & 1080.952 & 994.315 & 909.184 & 870.845 & 202.665 & 818.539 & 9678.492 \\
12 & 5192.053 & 982.723 & 768.211 & 777.985 & 363.490 & 923.699 & 1129.407 & 10137.568 \\
13 & 5685.900 & 598.837 & 752.744 & 660.163 & 1007.248 & 269.102 & 1526.953 & 10500.947 \\
14 & 2366.639 & 1089.153 & 451.300 & 2585.726 & 1878.995 & 817.197 & 2374.798 & 11563.808 \\
\hline
\end{tabular}

A RB needs to be identified for the case building for the evaluation of energy-saving potential and the improvement of occupant behavior. The buildings with less total energy consumption (i.e. the sum of the seven main end-use loads) than the case building in cluster_4 were considered to be RB candidates. In order to provide reliable information for the case building occupants, the RB was defined as the most similar building to the case building in terms of building-related parameters. The Euclidean distance was used to determine the similarity. Various building-related parameters were captured from the database to calculate the Euclidean distance. Among these parameters, five are categorical parameters and are transformed into [0, 1], as shown in Table 11.

Table 11

Transformation of categorical parameters

\begin{tabular}{ccccccc}
\hline Categorical parameters & \multicolumn{2}{c}{$\mathrm{CO}$} & & HT & \multicolumn{2}{c}{$\begin{array}{c}\text { Energy sources by usage } \\
\text { (SH, WH, KIT) }\end{array}$} \\
\hline Value & wood & non-wood & apartment & detached house & Electric & non-electric \\
Transformation value & 0 & 1 & 0 & 1 & 0 & 1 \\
\hline
\end{tabular}

Table 12 shows the building-related parameters of the RB candidate buildings and the case building. 


\section{Table 12}

Building-related parameters of RB candidate buildings and the case building

\begin{tabular}{|c|c|c|c|c|c|c|c|c|c|c|c|c|c|}
\hline \multirow{2}{*}{ No. } & \multirow{2}{*}{ NO } & \multirow{2}{*}{ FA } & \multirow{2}{*}{ HLC } & \multirow{2}{*}{ ELA } & \multirow{2}{*}{$\mathrm{CO}$} & \multirow{2}{*}{ HT } & \multicolumn{3}{|c|}{ Energy sources by usage } & \multirow{2}{*}{$\mathrm{T}$} & \multirow{2}{*}{$\mathrm{V}$} & \multirow{2}{*}{$\mathrm{RH}$} & \multirow{2}{*}{ RA } \\
\hline & & & & & & & SH & WH & KIT & & & & \\
\hline 1 & 4 & 112 & 2.04 & 4.385 & 1 & 1 & 1 & 0 & 0 & 15.1 & 2.1 & 73 & 12.3 \\
\hline 2 & 4 & 141.6 & 1.79 & 0.77 & 0 & 1 & 0 & 0 & 0 & 12.8 & 4.3 & 74 & 11.7 \\
\hline 3 & 2 & 185.9 & 1.87 & 0.35 & 1 & 1 & 1 & 1 & 1 & 8.8 & 3.6 & 68 & 12.6 \\
\hline 4 & 4 & 115 & 2.61 & 6.365 & 0 & 1 & 0 & 1 & 1 & 16.9 & 2.5 & 66 & 12.6 \\
\hline 5 & 2 & 87.05 & 0.83 & 1.06 & 1 & 0 & 1 & 1 & 1 & 8.8 & 3.6 & 68 & 12.6 \\
\hline 6 & 2 & 135 & 1.7 & 3.9 & 1 & 1 & 0 & 0 & 0 & 17.2 & 2.8 & 66 & 13.1 \\
\hline 7 & 4 & 160.6 & 1.84 & 2.20 & 0 & 1 & 1 & 1 & 1 & 11.8 & 4.2 & 72 & 11.8 \\
\hline $8^{*}$ & 2 & 128.3 & 1.69 & 0.6 & 0 & 1 & 0 & 1 & 1 & 8.8 & 3.6 & 68 & 12.6 \\
\hline
\end{tabular}

* The case building.

Again, the min-max normalization was applied in order to help prevent attributes with large ranges from outweighing those with comparatively smaller ranges. After normalization, the Euclidean distance between each candidate building and the case building was calculated; and the building with the smallest distance, i.e. No.3 building in Tables 12 and 10, was identified as the RB. For comparison, Table 13 shows the main end-use loads in the case building and the RB.

\section{Table 13}

Comparison of end-use data between the case building and RB (Unit: MJ per capita per year)

\begin{tabular}{ccccccccc}
\hline Building & SHW & LIGHT & KITCH & REFRI & E\&I & H\&S & OTHER & Sum \\
\hline Case building & 3882.699 & 582.052 & 250.6 & 1541.394 & 1799.53 & 621.743 & 336.592 & 9014.61 \\
RB & 1464.821 & 287.523 & 936.88 & 924.793 & 1958.911 & 504.171 & 845.352 & 6922.45 \\
\hline
\end{tabular}

Table 13 shows that the sum of energy consumption in the case building is evidently higher than that in the RB. Further, user activities in the case building caused significantly higher energy consumption in SHW, LIGHT, REFRI, and H\&S than that of the RB. This indicates that, in comparison with buildings with similar occupant behavior and building-related parameters, energy-saving potential still exists for the case building. That means energy consumption may be considerably reduced through modifying occupant behavior related to SHW, LIGHT, REFRI, and H\&S. It should be noted that energy consumption in REFRI in cluster_4 is also medium high when comparing with the other three clusters. This implies the energy-saving potential of REFRI-related behavior is comparatively higher than the potential of the others, and thus deserves extra attention.

Additionally, energy-saving potential in the case building can be identified as the energy consumption difference between the two buildings, i.e. $9014.610-6922.450=$ 
2092.161 MJ per capita per year.

\section{3. Association rule mining (ARM) in the case building}

Based on the information obtained from cluster-then-classification and RB identification, the ARM was then performed to find all the associations among the end-use loads at both levels. Accordingly, energy-inefficient specific occupant behavior will be determined and then energy-saving recommendations for modifying activities can be provided for the case building occupants.

After experimenting with various combinations of support and confidence values, a support of $50 \%$ and a confidence of $80 \%$ were set as minimum thresholds. Such thresholds mean that, for each generated association rule, at least $50 \%$ of all the data records under analysis contain both premise and conclusion; and the probability that a premise's emergence leads to a conclusion's occurrence is $80 \%$ or more. In addition, the minimum threshold of lift value was set 1 to find positive correlations. Such mining generated 756 rules, many of which are obvious and uninteresting; and truly interesting rules need to be further identified based on domain knowledge. Fifteen association rules between household appliances were selected for demonstration purposes, as shown in Table 14. It should be mentioned that most obtained associations are between attributes in the LOW range (i.e. low energy consumption), while clearly the associations in the HIGH range (i.e. high energy consumption) may provide more useful information on energy conservation. This also indicates that the attributes involved in the obtained rules have a skewed distribution toward the LOW range, and may be ascribed to the high degree of building occupants' energy-saving consciousness. Moreover, due to the availability of the data source, daily data was used for ARM instead of hourly data; and thus the obtained rules do not necessarily indicate that user activities in the premises and conclusions occur simultaneously. Therefore, the actual occupant behavior patterns should also be taken into consideration when using these rules in practice.

\section{Table 14}

Selected association rules $\left(\min \_s u p^{a^{*}}=50 \%\right.$, min_conf $^{\mathrm{b}^{*}}=80 \%$, min_lift $\left.{ }^{c^{*}}=1\right)$

\begin{tabular}{|c|c|c|c|c|c|}
\hline No. & Premise & Conclusion & Sup. & Conf. & Lift \\
\hline Rule 1 & Living room outlet [LOW] & OTHER [LOW] & $54 \%$ & $98 \%$ & 1.49 \\
\hline Rule 2 & Heating boiler [HIGH] & REFRI [HIGH] & $51 \%$ & $94 \%$ & 1.12 \\
\hline Rule 3 & Lamp 1F [LOW] & LIGHT [LOW] & $59 \%$ & $96 \%$ & 1.33 \\
\hline Rule 4 & Washing machine $2 \mathrm{~F}$ [LOW] & H\&S [LOW] & $76 \%$ & $97 \%$ & 1.25 \\
\hline Rule 5 & Dishwasher [LOW] & KITCH [LOW] & $74 \%$ & $99 \%$ & 1.26 \\
\hline Rule 6 & Vacuum cleaner [LOW] & H\&S [LOW] & $67 \%$ & $84 \%$ & 1.07 \\
\hline Rule 7 & Microwave, toaster, coffee [LOW] & KITCH [LOW] & $66 \%$ & $81 \%$ & 1.04 \\
\hline Rule 8 & TV (master bedroom 2F) [LOW] & Lamp 2F [LOW] & $66 \%$ & $87 \%$ & 1.10 \\
\hline Rule 9 & TV (other rooms) [LOW] & LIGHT [LOW] & $51 \%$ & $81 \%$ & 1.11 \\
\hline
\end{tabular}




\begin{tabular}{|c|c|c|c|c|c|}
\hline Rule 10 & Video [LOW] & Table lamp [LOW] & $52 \%$ & $84 \%$ & 1.02 \\
\hline Rule 11 & Lamp 1F [LOW] & Table lamp [LOW] & $52 \%$ & $84 \%$ & 1.02 \\
\hline Rule 12 & TV (Standby Power) [HIGH] & Ventilator [HIGH] & $55 \%$ & $100 \%$ & 1.82 \\
\hline Rule 13 & Phone [LOW] & Boom box [LOW] & $57 \%$ & $90 \%$ & 1.06 \\
\hline Rule 14 & TV (dining room) [LOW] & Boom box [LOW] & $51 \%$ & $85 \%$ & 1.01 \\
\hline Rule 15 & TV (other rooms) [LOW] & Boom box [LOW] & $54 \%$ & $86 \%$ & 1.02 \\
\hline
\end{tabular}

The results of the cluster analysis show that the case building was grouped into cluster_4, which was characterized as the building group with high energy consumption in OTHER, medium high energy consumption in REFRI and E\&I. Hence, association rules involving OTHER, REFRI and E\&I are the most important and deserve more attention. Accordingly, two rules, i.e. Rule 1 and Rule 2 in Table 14, were found among all the obtained rules and discussed as follows:

Rule 1 shows that living room outlet and OTHER have a strong positive association with a confidence of $98 \%$ and a lift of 1.49 . From this rule, it can be inferred that, in this building, the electricity load increase in living room outlet would quite possibly lead to the increase in OTHER. This indicates that, among all the unclear items included in OTHER, removable electrically-operated devices connecting to the living-room power plugs deserve more attention than other devices. Therefore, building owners could easily identify these devices and then manage to modify their usage to reduce energy consumption.

Rule 2 shows that heating boiler has a strong positive association with REFRI with a confidence of $94 \%$ and a lift of 1.12. Given that the daily energy consumption of the heating boiler is mainly impacted by occupant presence and outdoor air temperature, this rule implies that, two factors (i.e. both a longer stay time of occupants and a lower outdoor air temperature) possibly cause a higher energy consumption of refrigerators. With regard to the first factor, it sounds reasonable since a longer stay time of occupants tends to increase the refrigerator usage, thereby increasing the energy consumption. With regard to the second factor, it seems unreasonable since a low outdoor air temperature normally causes a relatively low indoor air temperature in a detached house without central HVAC systems, thereby decreasing the energy consumption of refrigerators. A possible explanation for this is that the building occupants had high thermal comfort requirements in cold days; and preferred to a high indoor air temperature by increasing the boiler thermostat setting or using kerosene space-heaters. In order to justify the assumption, the pattern relating mean daily kitchen air temperature ${ }^{1}$ to mean daily outdoor air temperature was plotted, as shown in Fig. 9. A trend line was then drawn to find out whether the kitchen air

\footnotetext{
${ }^{1}$ In this building, both the kitchen and the living room are in the first floor, and there are no partitions between them. Hence, they have the same indoor air temperature and the living room air temperature was used in this figure.
} 
temperature increased or decreased in relation to outdoor air temperature. Clearly, a downward trend in mean daily kitchen air temperature following the increase of mean daily outdoor air temperature can be observed, which is in accordance with the assumption.

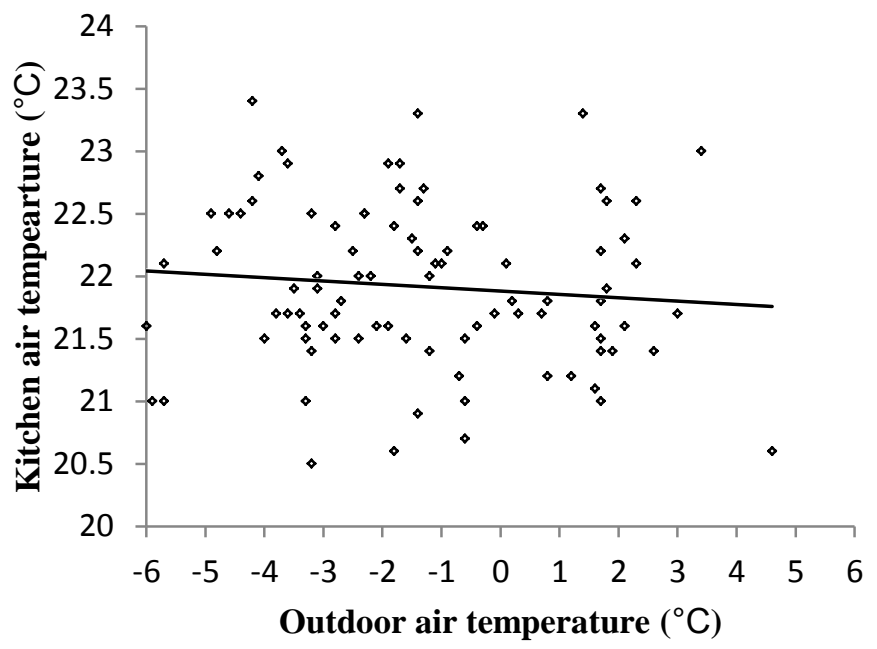

Fig.9. Mean daily air temperature in kitchen vs. mean daily outdoor air temperature (winter, 2003)

Therefore, a trade-off between human thermal comfort and building energy consumption is necessary for the owners, since an appropriate decrease of indoor thermostat settings in cold days results in an energy-consumption reduction in both space heating and refrigerators.

Further, the comparison between the $\mathrm{RB}$ and the case building shows that user activities in the case building caused significantly higher energy consumption in SHW, LIGHT, REFRI, and H\&S than those in the RB. Hence, rules associating with these four attributes also deserve extra attention. At the same time, in order to provide more comprehensive recommendations for energy-efficient behavior, rules associating with other end-use loads were also analyzed in this study. Eventually, thirteen interesting rules (i.e. Rules 3 to 15 in Table 14) were selected and discussed as follows.

Similar to Rule 1, Rules 3, 4, and 5 show that lamp $1 F$, washing machine $2 F$ and dishwasher have a strong positive association with LIGHT, H\&S, and $\mathrm{KITCH}$, respectively. Rules 6 and 7 show that vacuum cleaner, and microwave, toaster, coffee have a positive association with $\mathrm{H} \& \mathrm{~S}$ and $\mathrm{KITCH}$, respectively. Therefore, comparing with other appliances associating with LIGHT, H\&S, and KITCH, the building occupants should pay more attention to the use of lamps in the first floor, washing machines in the second floor, and dishwashers, since activities related to these appliances could have a major influence on the corresponding main end-use loads. At the same time, the use of vacuum cleaners, microwave ovens, toasters, and coffee machines also deserve some attention, though their associations with $\mathrm{H} \& \mathrm{~S}$ and $\mathrm{KITCH}$ are weaker than washing machine $2 F$ and dishwasher.

Rule 8 shows that $T V$ (master bedroom $2 F$ ) has a positive association with lamp $2 F$ 
with a confidence of $87 \%$ and a lift of 1.10 . From this rule, it can be inferred that the usage of $T V$ (master bedroom $2 F$ ) would quite possibly lead to the usage of lamp $2 F$. This may have occurred since the building occupants always turned the lights on when they were watching TV. An effective way of reducing energy consumption in this building is to watch TV with dim light.

Rules 9 to 11 can be explained in the same way as Rule 8 and similar recommendations can be provided.

An unexpected result was that TV (Standby Power) and Ventilator have a strong positive association with a confidence of $100 \%$ and a lift of 1.82, as shown in Rule 12 . Clearly the standby power of TVs and ventilators have the same trend of variation. This may have occurred since the building occupants would turn off the TVs and switch off the ventilators when the building was empty. However, standby power is commonly unnecessary and still accounts for energy cost. Therefore, TVs should be completely turned off or unplugged when they are not used. Furthermore, the wasted standby power of TVs is very small, but the sum of standby use consumed by all house appliances, such as microwave ovens, air conditioners, power adapters for laptop computers and other electronic devices, becomes significant. Standby power accounts for around 5-10\% of residential electrical energy use in most developed countries; and continues to increase in developing countries [21]. Hence, it is meaningful to help building owners to realize the importance of reducing standby power consumption, and feasible recommendations should also be provided for them. For example, a switchable power strip can be used for multiple devices, such as VCRs, DVD players, TVs, and computers, so that these appliances can be unplugged conveniently with one action.

Rules 13 to 15 show that phone, TV (dining room) and TV (other rooms) have a positive association with boom box. This indicates that, among all the appliances included in E\&I, boom boxes was used in comparatively high frequency and deserve extra attention.

Moreover, indoor and outdoor parameters were also included in this ARM model. Associations between indoor/outdoor parameters and household appliances can assist in understanding the factors influencing occupant behavior. In order to demonstrate such associations, six rules were selected and shown in Table 15.

\section{Table 15}

Selected association rules between indoor/outdoor parameters and household appliances $($ min_sup $=50 \%$, min_conf $=80 \%$, min_lift $=1$ )

\begin{tabular}{|c|c|c|c|c|c|}
\hline No. & Premise & Conclusion & Sup. & Conf. & Lift. \\
\hline Rule 1 & Master bedroom $(2 \mathrm{~F})$ temperature $[\mathrm{HIGH}]$ & Microwave, toaster, coffee [LOW] & $58 \%$ & $83 \%$ & 1.02 \\
\hline Rule 2 & Living room humidity [LOW] & Microwave, toaster, coffee [LOW] & $55 \%$ & $86 \%$ & 1.06 \\
\hline
\end{tabular}


Rule 3 Outdoor relative humidity [LOW]

Rule 4 Outdoor air temperature [LOW]

Rule 5 Outdoor air velocity [LOW]

Rule 6 Living room humidity [LOW]
Microwave, toaster, coffee [LOW]

H\&S [LOW]

$\mathrm{H} \& \mathrm{~S}$ [LOW]

H\&S [LOW]

\begin{tabular}{lll}
$57 \%$ & $87 \%$ & 1.07 \\
$54 \%$ & $88 \%$ & 1.12 \\
$59 \%$ & $82 \%$ & 1.05 \\
$57 \%$ & $90 \%$ & 1.15 \\
\hline
\end{tabular}

Rules 1 to 3 show that master bedroom ( $2 F)$ temperature (HIGH), living room humidity, and outdoor relative humidity have a positive association with microwave, toaster and coffee. This indicates that a high master bedroom temperature, as well as a low living room or outdoor relative humidity, tends to decrease the usage of microwave ovens, toasters, and coffee machines. A possible explanation for this is that the increase in indoor air temperature, or the decrease in indoor/outdoor relative humidity, causes the occupants to lose their appetite to some extent.

Rules 4 to 6 show that outdoor air temperature, outdoor air velocity, and living room humidity have a positive association with $H \& S$. This indicates that the decrease in outdoor air temperature/velocity, and living room humidity tends to reduce the likelihood that occupants do housework such as cleaning and washing. It can be inferred that both local climatic conditions and indoor microclimate may have an impact on occupant behavior relating to housework. For example, the increase of outdoor air velocity may deteriorate indoor sanitary conditions (dust accumulation), thereby increasing the usage of vacuum cleaners and other sanitary appliances.

In addition, based on all the generated rules, it was found that six attributes, as shown in Table 16, have no association with the remaining attributes.

\section{Table 16}

Attributes without associations with the remaining attributes

\begin{tabular}{ccc}
\hline No. & Appliances & Indoor parameters \\
\hline 1 & Total energy consumption & Living room temperature \\
2 & I\&E & Bedroom (1F) temperature \\
3 & Bidet & \\
4 & IH heater & \\
\hline
\end{tabular}

The fact that these attributes have no association with the other attributes implies that, in this building, they are independent. There are two possible reasons for these attributes' independence: for total energy consumption and I\&E, they may be decided by the holistic effects of various user activities, instead of associating with some certain activity. For the other four attributes, their values may be purely random or remain relatively stable in the whole winter and thus no association with other attributes can be found. Such information can help building owners to make intelligent decisions when modifying their behavior. 


\section{Conclusions}

A methodology for identifying and improving occupant behavior in existing residential buildings is developed. End-use loads of various household appliances were mapped onto corresponding occupant behavior, and were used to deduce user activities indirectly in this study. Specifically, these end-use loads were divided into two levels (main and sub-category), and thus correspond to two-level activities, i.e. general and specific occupant behavior.

In order to demonstrate its applicability, this methodology was applied to a group of residential buildings located in six different districts of Japan. Field surveys on energy-related data and other relevant information were carried out, and then a database was developed. A building with the most comprehensive household appliances was selected as the case building and the remaining buildings were used as related buildings. Data pre-processing was performed for the related buildings and they were grouped into four clusters by using K-means algorithm. The characteristic of occupant behavior in each cluster was analyzed. Base on these clusters, a decision tree was generated and its accuracy was evaluated as $80 \%$. In terms of the decision tree, the case building was predicted to belong to cluster_4. A reference building was identified in the same cluster as the case building. Consequently, the case building was compared with buildings in the other clusters and the reference building to determine energy-inefficient general behavior. Also, its energy-saving potential was identified as 2092.161 MJ per capita per year. Moreover, association rules were mined based on the data of the case building in winter in 2003, given the seasonality of occupant behavior. A number of interesting rules were found, and associations and correlations between different user activities were discovered. According to these rules, specific recommendations for highlighting energy-saving opportunities were provided for the building occupants.

Considering the diversity of specific occupant behavior, the determination of energy-inefficient general occupant behavior can narrow down the scope of identification of energy-inefficient specific occupant behavior, and thus can help occupants to quickly find the generated association rules, as well as specific behavior, which deserve more attention. Also, such information is extracted from the real measured data and covers almost all energy-related behavior. With such information, building occupants can then clearly understand their actual behavior patterns, and easily focus on the energy-inefficient behavior needing to be modified. Therefore, the main advantage of the proposed methodology lies in its high efficiency of occupant behavior improvement. Moreover, the identification of energy-inefficient general behavior in this study is mainly based on the comparison with other similar buildings; this can help building owners to be aware of avoidable energy waste caused by their behavior, and motivate them to modify their activities accordingly.

The application of this proposed methodology to Japanese residential buildings in this 
paper has clearly proved that this methodology is more efficient and rational than the traditional methods, i.e. energy saving education method and building simulation method. However, further study is still necessary and the main focus of future research should be placed on identifying appropriate database sizes and the number of clusters, improving the accuracy of generated decision tree. These measures have a strong influence on characterizing the occupant behavior in all the investigated buildings and cluster attribution of the case building. In addition, it is noted that using daily end-use loads in the case building to mine associaton rules and provide recommendations for occupants is not sufficient. This is because user activities in the premises and conclusions of association rules may not occur simultaneously. In order to overcome this limitation, hourly (or less than one hour, such as 15 minutes) end-use loads of various household appliances should be measured and used in association rule mining.

\section{Acknowledgements}

The authors would like to express their gratitude to the Public Works and Government Services Canada, and Concordia University for the financial support. The authors also wish to thank the reviewers for their valuable comments.

\section{References}

[1] Swan LG, Ugursal VI. Modeling of end-use energy consumption in the residential sector: A review of modeling techniques. Renewable and Sustainable Energy Reviews 2009; 13(8): $1819-1835$.

[2] Chen SQ, Li NP, Guan J, Xie YQ, Sun FM, Ni J. A statistical method to investigate national energy consumption in the residential building sector of China. Energy and Buildings 2008; 40(4): 654-665.

[3] Tanatvanit S, Limmeechokchai B, Chungpaibulpatana S. Sustainable energy development strategies: implications of energy demand management and renewable energy in Thailand. Renewable and Sustainable Energy Reviews 2003; 7(5): 367-395.

[4] Ruijven BV, Vries BD, Van Vuuren DP, Van der Sluijs JP. A global model for residential energy use: Uncertainty in calibration to regional data. Energy 2010; 35(1): 269-282.

[5] Shimoda Y, Asahi T, Taniguchi A, Mizuno M. Evaluation of city-scale impact of residential energy conservation measures using the detailed end-use simulation model. Energy 2007; 32(9): $1617-1633$.

[6] Kenisarin M, Kenisarina K. Energy saving potential in the residential sector of Uzbekistan. Energy 2007; 32(8): 1319-1325.[7] Yu Z, Fung BCM, Haghighat F, Yoshino H, Morofsky E. A systematic procedure to study the influence of occupant behavior on building energy consumption. Energy and Buildings 2011; 43(6): 1409-1417

[8] Ouyang JL, Hokao K, Energy-saving potential by improving occupants' behavior in urban residential sector in Hangzhou City, China. Energy and Buildings 2009; 41(7): 711-720.

[9] Al-Mumin A, Khattab O, Sridhar G. Occupants' behavior and activity patterns influencing the energy consumption in the Kuwaiti residences. Energy and Buildings 2003; 35(6): 549-559.

[10] Zmeureanu R, Fazio P, DePani S, Calla R. Development of an energy rating system for 
existing houses. Energy and Buildings 1999; 29(2): 107-119.

[11] Han JW, Kamber M. Data Mining Concepts and Techniques. 2nd ed. San Francisco: Elsevier Inc; 2006.

[12] Yu Z, Haghighat F, Fung BCM, Yoshino H. A decision tree method for building energy demand modeling, Energy and Buildings (2010); 42(10): 1637-1646.

[13] Rapid - I - RapidMiner. http://rapid-i.com/content/view/181/190/

[14] Davies DL, Bouldin DW. A cluster separation measure, IEEE Trans Pattern Anal Mach Intelligence 1979; 1: 224-227.

[15] Quinlan JR. Induction of decision trees. Machine Learning 1986; 1: 81-106.

[16] L.Breiman, J.H.Friedman, R.A.Olshen, C.J.Stone, Classification and Regression Trees. 2nd ed. Belmont: Wadsworth Inc.; 1984.

[17] J.R. Quinlan. C4.5 Programs for Machine Learning. 1st ed. San Mateo: Morgan Kaufmann; 1993.

[18] Association Rule Mining with WEKA.

http://maya.cs.depaul.edu/ Classes/Ect584/Weka/associate.html

[19] Murakami S, Akabayashi S, Inoue T, Yoshino H, Hasegawa K, Yuasa K, Ikaga T, Energy consumption for residential buildings in Japan, Architectural Institute of Japan, Maruzen Corp., 2006 (http://tkkankyo.eng.niigata-u.ac.jp/HP/HP/database/index.htm).

[20] Helsel DR, Hirsch RM. Statistical methods in water resources. U.S. department of the interior 2002

[21] Standby Power, Frequently Asked Questions (FAQs). http://standby.lbl.gov/faq.html 